ISSN 0257-1439

Revista Costarricense de Psicología, Vol. 29, n. ${ }^{\circ} 43,2010$, 57-74.

DOI: http://dx.doi.org/10.22544/rcps.v29i43.05

\title{
Ejercicio físico, funcionamiento cerebral e imagen corporal
}

\author{
Physical Exercise, Brain Functioning, and Body Image
}

José Moncada Jiménez *

Universidad de Costa Rica

(recibido 1 de abril de 2008; aceptado 16 de noviembre de 2009)

\begin{abstract}
Resumen
La imagen corporal es un componente del yo, y se ha estimado que una cantidad importante de mujeres y hombres de diversas edades, razas y orígenes étnicos poseen insatisfacción de su imagen corporal. Por lo tanto, el tema de la imagen corporal preocupa a la opinión pública ya que se le ha relacionado con conductas tales como los desórdenes alimenticios. La anorexia y la bulimia constituyen las enfermedades más importantes. La presente revisión resume las relaciones entre las áreas cerebrales involucradas en la generación y evaluación del cuerpo humano, y la manera en que el ejercicio físico podría influir en esas zonas para producir una imagen corporal objetiva y precisa. Recientemente, se ha identificado el área corporal extraestriada (ACE) como la estructura cerebral primordialmente responsable del reconocimiento del cuerpo humano. Se deben realizar más estudios que involucren el ACE. En esta revisión también se discuten aspectos relacionados con el metabolismo cerebral en reposo y durante el ejercicio físico y su relación con cambios en la estructura y funcionamiento cerebral. Finalmente, se presenta un resumen de los estudios sobre el efecto del ejercicio aeróbico y contra resistencia en la imagen corporal.
\end{abstract}

Palabras clave: imagen corporal, sistema del yo, cerebro, metabolismo, ejercicio

\begin{abstract}
Body image is a component of the self. It has been estimated that a large group of males and females of heterogeneous ages and racial/ethnic backgrounds are discontent with their body image. Therefore, public opinion shows concern on the topic of body image since it has been related to eating disorders, especially anorexia and bulimia. This review summarizes the relationship between the brain areas involved in the generation and evaluation of body image and the way exercise might impact those areas to generate an accurate and objective body image. The extrastriate body area (EBA) in the brain has been recently identified as the structure primarily involved in the recognition of the human
\end{abstract}

* Escuela de Educación Física y Deportes, Universidad de Costa Rica. Correspondencia. Correo electrónico: jose.moncada@ucr.ac.cr Nota: El Consejo Editorial de la Revista Costarricense de Psicología aclara que, debido a un error involuntario, este artículo se excluyó del Vol. 28, No. 41-42, 2009, por lo cual el trabajo se incluyó para su publicación final en el Vol. 29, No. 43, 2010. Se hace la aclaración basado en nota CPPCR-CERCP-101-2008.

ISSN 0257-1439

(C) Colegio Profesional de Psicólogos de Costa Rica

http://www.revistacostarricensedepsicologia.com/ 
body. Further research on the EBA has to be carried out. Also, brain metabolism at rest and during exercise and its relationship with brain changes in structure and function are discussed. Finally, a summary of the effects of aerobic and resistance exercise training on body image is presented.

Keywords: body image, self-system, brain, metabolism, exercise

La actividad física y el ejercicio físico son considerados conductas o comportamientos, y se ha demostrado que poblaciones desarrolladas y en vías de desarrollo no alcanzan a cumplir con los estándares mínimos que les permita reducir el riesgo de desarrollar una amplia gama de enfermedades, incluyendo disturbios físicos y psicológicos (Buckworth \& Dishman, 2002; Pate et al., 1995; United States Department of Health and Human Services, 1996).

Una amplia serie de investigaciones han utilizado modelos que han permitido la identificación de algunos determinantes de la actividad física, por ejemplo, la pérdida de peso, la autoeficacia, el apoyo social, la autorregulación, la percepción de la salud, la influencia social o de pares y la imagen corporal (Bryan \& Rocheleau, 2002; Deforche, Bourdeaudhuij, Tanghe, Hills, \& De Bode, 2004; Dishman et al., 2004; Netz \& Raviv, 2004; Dallow \& Anderson, 2003; Rovniak, Anderson, \& Winett, 2002; Trost, Kerr, Ward, \& Pate, 2001; Trost, Saunders, \& Ward, 2002).

La presente revisión se concentrará en los potenciales mecanismos por medio de los cuales el ejercicio físico podría influenciar regiones cerebrales encargadas de percibir, producir y analizar la figura humana y la imagen corporal. Este tema es relevante ya que se ha demostrado que los medios de comunicación afectan la percepción de la imagen corporal en los adolescentes y, a su vez, el grado de actividad física (Katzmarzyk \& Davis, 2001; Taveras, Rifas-Shiman, Field, Frazier, Golditz, \& Gillman, 2004). Es más, de acuerdo con la teoría de comparación social, la percepción de la imagen corporal sería un proceso innato por medio del cual una persona recolecta información relacionada con un atributo. Por lo tanto, e.g., los medios de comunicación masiva tienen el potencial de influir en la decisión personal acerca de cómo debería lucir un cuerpo "modelo" o "ideal".

En Costa Rica, y en otros países, autores como Cash y Deagle (1997), Feingold y Mazzella (1998), Gokee-La Rose, Dunn y Tantleff-Dunn (2004), Hargreaves y Tiggemann (2004), Mayville, Katz, Gipson y Cabral (1999), McLaren y Kuh (2004), Scully, Kremer, Meade, Graham y Dudgeon (1998) han reportado problemas nutricionales, desórdenes, distorsiones y disturbios dismórficos, ansiedad relacionada con la imagen corporal, insatisfacción corporal, y deseo por la delgadez en hombres y mujeres de diferentes edades y grupos étnicos, sin embargo, estos problemas son más comunes en personas con sobrepeso y obesidad. Por ejemplo, de acuerdo con Hausenblas y Symons (2001), las investigaciones previas de 1972 a 1996 han indicado una alta tasa de insatisfacción corporal en la población estadounidense, en donde las mujeres tuvieron tasas más altas (23-56\%) en comparación con los hombres (15-43\%). Desde este contexto, el estudio de la imagen corporal en general, así como el estudio de los desórdenes relacionados con la imagen corporal se torna relevante debido a que las personas con insatisfacción corporal (e.g., personas con anorexia nerviosa) muestran tasas de suicidio más altas que la población general (Hausenblas \& Symons, 2001). Otros aspectos relevantes se relacionan con gastos mayores en cirugía cosmética, productos dietéticos, suplementos para reducir peso, y la aparición de otras enfermedades mentales (Pompili, Mancianelli, Girardi, Ruberto, \& Tatarelli, 2004). 
Hausenblas y Symons (2001) presentan una definición de imagen corporal básica, y la describen como un constructo psicológico que se refiere a "la representación interna de su apariencia externa" (p.323). Otros investigadores se han referido al mismo constructo con diferentes nombres, por ejemplo, esquema corporal, catexis corporal, conciencia corporal, y apropiación corporal (Skrinar, Bullen, Cheek, Mc Arthur, \& Vaughan, 1986; Tucker \& Maxwell, 1992). Algunos autores han indicado que la imagen corporal se refiere a "una representación consciente del cuerpo así como al conocimiento conceptual del mismo" (Coslett, 1998, p. 528); mientras que el esquema corporal se refiere a una representación abstracta que cada persona percibe de su cuerpo en el espacio, en tiempo real, en línea, y que se deriva de estímulos sensoriales, incluyendo movimientos musculares, propioceptivos, cutáneos, vestibulares, táctiles, visuales y auditivos (Vogeley, Kurthen, Falkai, \& Maier, 1999). Para propósitos de la presente revisión se utilizará la definición de imagen corporal de Hausenblas y Symons (2001).

De acuerdo con Vogeley et al. (1999), el modelo o sistema del yo, es un "constructo teórico que comprende características esenciales que son accesibles por introspección” (p. 344), las cuales incluyen experiencias de apropiamiento, perspectiva y unidad, ya que la imagen corporal es un componente del sistema del yo, está formada por múltiples percepciones que se pueden relacionar con una salud mental positiva general (Buckworth \& Dishman, 2002). Sin embargo, esto no podría ser necesariamente cierto, tal y como se discutirá más adelante.

Diversos investigadores han relacionado positivamente el ejercicio físico regular con la imagen corporal (Ahmed, Hilton, \& Pituch, 2002; Kirkcaldy, Shephard, \& Siefen, 2002; Perry, Rosenblatt, Kempner, Feldman, Paolercio, \& van Bemden, 2002). Sin embargo, el ejercicio excesivo, unido a los desórdenes alimentarios, se ha relacionado con una imagen corporal negativa.

Por lo tanto, el propósito principal del presente trabajo es describir los mecanismos potenciales por medio de los cuales el ejercicio físico podría afectar la imagen corporal. Como se ha mencionado anteriormente, existen múltiples factores que están involucrados en la interpretación de la imagen corporal, por lo que el enfoque de esta revisión será sobre la manera en que el cerebro percibe las señales que "construyen" una imagen corporal humana. También se describirán los efectos del ejercicio físico o el entrenamiento en la actividad cerebral, para finalmente integrar y discutir la posible relación entre el ejercicio, el funcionamiento cerebral y la imagen corporal.

\section{Procesamiento visual del cuerpo humano}

De acuerdo con Goodale (2001), la función primordial de la vista es la de crear un modelo interno del mundo externo, el cual se convierte en la base perceptual de todos los pensamientos y las acciones motivadas por estímulos visuales. En la actualidad, se investiga acerca de las áreas o estructuras cerebrales responsables de procesar las señales visuales que construyen la imagen de un ser humano (Downing, Jiang, Shuman, \& Kanwisher, 2001).

El razonamiento que apoya la mayoría de las investigaciones en este campo se basa en el paradigma de que el cerebro está conformado por módulos que procesan información del yo selectivamente, incluyendo partes o segmentos corporales. Sin embargo, actualmente existe un debate acerca de si el cerebro procesa información de manera modular o "distribuida" (Cohen \& Tong, 2001). La teoría modular establece que tipos particulares de información se procesan y se presentan en el cerebro en módulos o segmentos específicos; mientras que la teoría de distribución indica que cualquier tipo de información, sin importar su naturaleza, es procesada por varias regiones del cerebro, y que cualquier parte del cerebro es capaz de representar información variada 
(Cohen \& Tong, 2001). Así, de acuerdo con estos investigadores, la mayoría de la evidencia apoya la teoría de los módulos ya que la teoría de la distribución no ha alcanzado los medios técnicos ni tecnológicos para que pueda estudiarse con profundidad. Por el momento, las técnicas más comúnmente conocidas, utilizadas en la investigación modular, han sido los potenciales relacionados con eventos (event related potential, ERP, por sus siglas en inglés), electroencefalografía (EEG), y recientemente, imágenes de resonancia magnética (magnetic resonance imaging, MRI, por sus siglas en inglés) e imágenes de resonancia magnética funcional (functional magnetic resonance imaging, fMRI, por sus siglas en inglés); sin embargo, esas técnicas no se han utilizado ampliamente en el estudio de la teoría de la distribución.

Un ejemplo tradicional de procesamiento de información de forma modular es la forma en que el cuerpo percibe sus diferentes partes. Esto ha sido gráficamente ilustrado por el homúnculo motor, un mapa deformado del cuerpo con el que supuestamente la corteza motora primaria interpreta el cuerpo (Helmuth, 2002) (Figura 1). Sin embargo, estudios recientes en monos sugieren que la corteza motora primaria no dibuja fielmente la superficie corporal, sino que más bien representa una imagen cerebral interna unida a percepciones subjetivas más que a señales sensoriales objetivas (Chen, Friedman, \& Roe, 2003; Eysel, 2003).
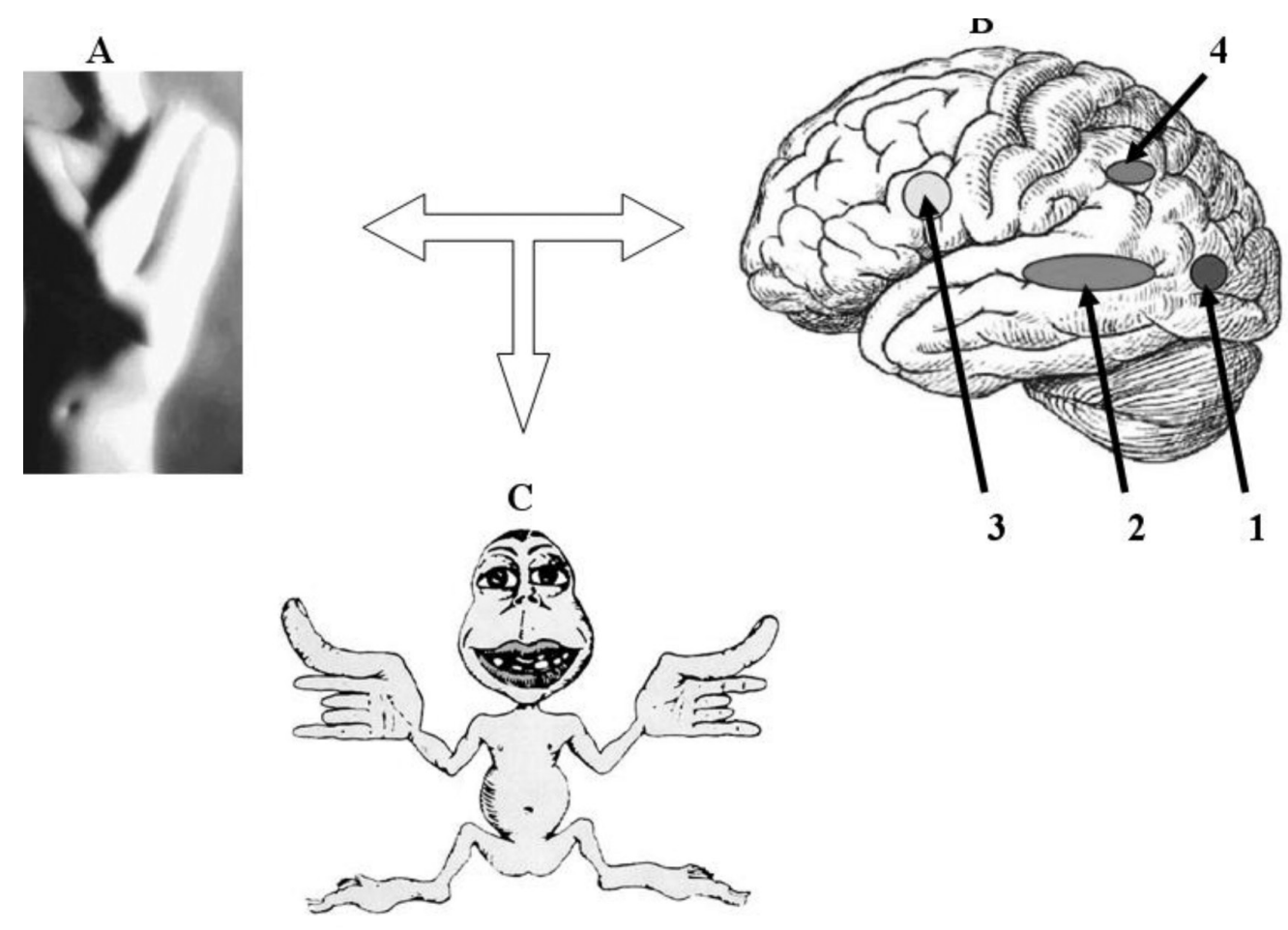

Figura 1. El reconocimiento de la imagen corporal (A) es un proceso complejo que requiere la integración visual, táctil, y de otros estímulos perceptuales en el cerebro (B). Las áreas de mayor sensibilidad percibida en las áreas corticales son exageradas en el dibujo completo del homúnculo humano (C). Las áreas corticales en el cerebro involucradas en el reconocimiento propio se numeran así: 1) ACE; 2) Sulcus temporal superior; 3) Área de Broca; y 4) Corteza parietal posterior. Fuentes: A) Umaña (2005); B) Jeannerod (2004); y C) Kandel y Mack (2003). 
Sin embargo, uno de los más significativos avances en esta área fue el descubrimiento realizado por Downing et al. (2001), quienes identificaron una región o módulo en la corteza occipitotemporal lateral derecha que respondía de manera diferenciada a fotografías estáticas de cuerpos humanos o partes del cuerpo. Los investigadores nombraron esa estructura el área corporal extraestriada (ACE, Downing et al., 2001, p. 2470) (Figura 1). Estos hallazgos se sustentaron en las imágenes obtenidas a través del fMRI, en donde se obtuvieron mayores respuestas eléctricas cerebrales en el 100\% de los sujetos que vieron las imágenes estáticas de los cuerpos humanos. Es más, la respuesta de $\mathrm{ACE}$ fue mayor al cuerpo humano completo que cuando se mostraron partes del cuerpo.

A pesar de este importante hallazgo, Downing et al. (2001) mencionan que no estaban completamente seguros de que una mayor respuesta del $\mathrm{ACE}$ indicara una preferencia definitiva por el cuerpo completo en comparación con las partes del cuerpo. Jeannerod (2004), indicó que el ACE podría explicar la sensación de propiedad o apropiación corporal, lo cual integraría correlaciones espaciales y temporales entre señales visuales y no visuales, táctiles y propioceptivas.

Siguiendo el estudio de Downing et al. (2001), Dohle, Kleiser, Seitz y Freund (2004), diseñaron una investigación para determinar en qué parte del cerebro interactuaba la imagen corporal percibida con la retroalimentación propioceptiva interna. Para comprender mejor el mecanismo involucrado, los investigadores utilizaron fMRI en seis sujetos quienes participaron en una maniobra de perturbación visual, que básicamente consistía en mantener la mano estática o en volver la mano con el dedo pulgar hacia abajo (movimiento de inversión). Dohle et al. (2004) concluyeron que la perturbación de la imagen corporal modificaba fuertemente la actividad de las cortezas visuales, donde la corteza visual primaria respondía preferentemente a las posturas estáticas de la mano en comparación con las posturas en movimiento. La relevancia de estos hallazgos radica en que se demostró que el movimiento puede ajustar la corteza visual de la misma manera que lo hace la atención; es decir, añadiendo información previa en una etapa anterior al procesamiento visual.

Además, se ha usado el N170, un potencial cerebral negativo que se dispara aproximadamente 170 ms después de haberse dado el estímulo en las áreas occipitotemporales laterales. Stekelenburg y de Gelder (2004) reportaron una mayor respuesta de los N170 durante las etapas iniciales de codificación de la información de la percepción del cuerpo humano. Este hallazgo sugiere que, cuando ocurre un procesamiento de la configuración del cuerpo humano, también existe una interacción entre los procesos de reconocimiento y los emocionales. Esto significa que la imagen corporal es el resultado de la interacción entre la identificación y la evaluación.

Los hallazgos de Downing et al. (2001) y Dohle et al. (2004) son especialmente relevantes puesto que en modelos animales se ha encontrado que la representación corporal en la superficie de la corteza cerebral se modifica por experiencia; es decir, el territorio cortical de una parte del cuerpo (y tal vez de todo el cuerpo) puede aumentarse con el entrenamiento (Kandel, 1998; Press, Taylor-Clarke, Kennett, \& Haggard, 2004). Esto significa que existe una plasticidad cerebral diferenciada, la cual involucra cambios en estructura y consecuentemente en funcionamiento; por ejemplo, en una situación de identificación de la imagen corporal. Esta hipótesis fue estudiada por Jenkins, Merzenich, Ochs, Allard y Guic-Robles (1990), quienes entrenaron monos para que ejecutaran una tarea manual $1 \mathrm{~h}$ /día durante tres meses. El diseño del estudio incluyó mediciones previas y posteriores al programa de ejercicios y se encontró que hubo un cambio significativo en la percepción corporal luego de tres meses de uso consistente de los dedos. 
Estas investigaciones indican que el reconocimiento de la imagen corporal es un proceso llevado a cabo en las estructuras cerebrales superiores que se ve influenciado por señales no solamente visuales, sino también por señales táctiles, constantes y permanentes. En términos prácticos, estos estudios son relevantes pues proporcionan una mejor comprensión acerca de cómo estas estructuras cerebrales específicas procesan diferentes tipos de señales en situaciones diversas, como por ejemplo, cuando nos miramos sin movernos frente a un espejo para evaluar nuestro cuerpo. Los estudios en humanos han demostrado la misma plasticidad en la corteza luego de una lesión y posterior entrenamiento (Elbert \& Rockstroh, 2004).

De acuerdo con Elbert y Rockstroh (2004), para que el cerebro pueda organizar su estructura y funcionamiento (i.e., representación cortical), antes se deben cumplir cuatro principios. Primero, una mayor estimulación de una parte del cuerpo aumenta su representación cortical y puede cambiar su orden topográfico. Estos cambios ocurren después de actividades perceptuales y conductuales, y el ejercicio físico podría ser una de éstas. Esta afirmación se basa en lo que Elbert y Rockstroh se refieren como el principio de que la práctica hace al maestro. El segundo principio es el de úselo o piérdalo, en el que una reducción o pérdida completa de señales aferentes lleva a una invasión de las zonas de representación ubicadas de manera adyacente al área deprivada de señales. Este principio se relaciona con la competencia por un espacio cortical, y este podría ser el caso de las personas sedentarias, quienes estarían causando un desbalance cerebral en el procesamiento de la información. Tercero, estímulos conductuales relevantes, sincrónicos de sitios adyacentes resultan, según Elbert y Rockstroh, en la integración de la representación cortical. Este principio llama conectadas y disparadas al mismo tiempo. Finalmente, estos investigadores indican que los cambios, únicamente, se observan en tareas conductuales relevantes y en respuesta a la práctica que es tan intensa y duradera que el cerebro sigue procesando aún cuando se duerma. Este concepto posee dos componentes que pueden ser relevantes para determinar el impacto del ejercicio en la imagen corporal. Primero, el ejercicio debe convertirse en una tarea relevante, de lo contrario, y de acuerdo con esta teoría, la intervención no produciría cambios en la estructura y funcionamiento del cerebro; y además, el ejercicio tiene que llevarse a cabo de manera intensa y por periodos prolongados de tiempo.

Con base en el trabajo de Elbert y Rockstroh, es posible sugerir que durante la distorsión de la imagen corporal, las personas son incapaces no solo de enviar información (señales) apropiada a la corteza visual, sino que también de brindar una retroalimentación positiva al cerebro. Por lo tanto, parece entonces que para percibir una imagen corporal positiva, una persona debería tocar su cuerpo constantemente mientras se mira frente al espejo. Esto implica que se debe entrenar a la persona a hacerlo y a recibir estímulos conductuales positivos (i.e., retroalimentación constante), de manera que las áreas corticales del cerebro puedan integrar toda la información para finalmente alcanzar cambios plásticos significativos. Sin embargo, esto también implica que este entrenamiento o práctica masiva tiene que llevarse a cabo por un periodo indeterminado de tiempo, pues no se han realizado investigaciones que apoyen la frecuencia de este tipo de intervención. Otras investigaciones con personas que han sufrido de derrame cerebral (accidente cerebrovascular) han utilizado regimenes de entrenamiento de tres a seis horas por día en 10 a 15 días sucesivos (Taub, Uswatte, \& Elbert, 2002). Se requiere mayor investigación para apoyar esta hipótesis, especialmente en personas aparentemente sanas.

La imagen corporal o el proceso de reconocimiento del cuerpo como una entidad unitaria posee un origen cognitivo y neurobiológico, el cual se puede dañar debido a lesiones o con drogas 
(Berlucchi \& Aglioti, 1997). Por ejemplo, existe el caso de una mujer que sufrió la ruptura de un aneurisma con una lesión concomitante en el lóbulo frontal en donde ésta percibía tener una "mano ajena" que intentaba ahogarla cuando nadaba en una piscina (Hari, Hänninen, Mäkinen, Jousmäki, Forss, Seppä, \& Salonen, 1998). Por otra parte, el procesamiento visual también puede verse alterado debido a una falta de respuesta en el cerebro, tal y como ha sido demostrado en estudios en sujetos sanos en los que se ha administrado un sedante y posteriormente se mide el nivel de oxígeno cerebral (Martin, Thiel, Joeri, Loenneker, Ekatrodramis, Huisman, Henning, \& Marcar, 2000); o en pacientes esquizofrénicos con deterioro de la corteza prefrontal (Berlucchi \& Aglioti, 1997; Vogeley et al., 1999).

También, se han descrito otras distorsiones de la imagen corporal al estudiar el lóbulo parietal posterior (Evans, 2004) y la amígdala derecha (Seeger, Braus, Ruf, Goldberger, \& Schmidt, 2002; Wagner, Ruf, Braus, \& Schmidt, 2003). Por ejemplo, en el síndrome de Alicia en el país de las maravillas, descrito en pacientes jóvenes con migraña de origen isquémico, encefalitis viral, y/o epilépticos, la persona describe episodios "donde se siente tan pequeña, como de un pie de altura" o "que [mi cuerpo] crece y crece cada vez más hasta que ocupa toda la habitación" (Evans, 2004, p. 625).

Recientemente, Northoff (2001) y Goodale (2001) integraron completamente el estado del conocimiento acerca de las diferentes áreas cerebrales y sus funciones específicas durante el procesamiento visual. De acuerdo con Northoff, la corteza somatosensorial construye una forma general del cuerpo al integrar estímulos táctiles y propioceptivos. El autor propone que la rama ventral del cerebro es la responsable de generar la representación perceptual del mundo visual y de los objetos que la habitan. Según Ehrsson, Spence y Passingham (2004), quienes llevaron a cabo una serie de experimentos y utilizaron fMRI para estudiar la actividad cerebral, la corteza premotora ventral está conectada anatómicamente a las áreas somatosensoriales y visuales en las áreas de la corteza parietal posterior y al área frontal, la cual la convierte en un área ideal para la representación multisensorial del cuerpo entero.

Además, el lóbulo parietal posterior (especialmente del hemisferio derecho), aparentemente proporciona un vínculo entre la forma corporal representada por la corteza somatosensorial y las coordinadas espaciales, lo que quiere decir que éstos vínculos producen un esquema espacial del cuerpo, es decir, la imagen corporal (Norhoff, 2001). Por último, la corteza insular juega un papel importante al conectar todas las estructuras previamente mencionadas con las regiones del sistema límbico involucradas en las funciones emocionales y viscerales. Esta organización permite a los individuos relacionar sus imágenes corporales con sus emociones (Norhoff, 2001). De manera similar, Goodale (2001) indicó que la región dorsal del cerebro interviene en la mediación del control visual de las acciones. Por lo tanto, se deduce que las percepciones de la imagen corporal (la capacidad para reconocer nuestro propio cuerpo) puede ser el resultado del procesamiento ventral del cerebro, mientras que la parte dorsal proporciona información sobre las características del cuerpo material o físico; por ejemplo, la ubicación espacial, el tamaño y la forma. De nuevo, la imagen corporal se convierte en un complejo proceso de identificación y evaluación.

\section{Efectos del ejercicio en la actividad cerebral}

En esta sección se discutirá el impacto de la modalidad, duración e intensidad del ejercicio en la actividad cerebral. Se presentarán los cambios metabólicos y plásticos encontrados en estudios realizados con modelos animales y humanos. También, se describirán brevemente las técnicas de medición utilizadas para registrar la actividad cerebral durante condiciones de ejercicio. 
De acuerdo con McCloskey, Adamo y Anderson (2001), las estructuras candidatas a someterse a cambios plásticos debidos al ejercicio y que muestran actividad durante la actividad física son el hipocampo, la corteza motora, y telencéfalo, las cuales han mostrado una mayor actividad neuronal durante el ejercicio. El supuesto en el que se basan las investigaciones en este campo es que el ejercicio mejora el funcionamiento cognoscitivo (McCloskey et al., 2001), posiblemente a través de aumentos en el flujo sanguíneo a las estructuras cerebrales superiores relacionadas con el procesamiento de las señales necesarias para generar movimientos. Por lo tanto, los aumentos en el flujo sanguíneo cerebral supuestamente suplen las necesidades metabólicas globales y locales en el cerebro, por ejemplo, las del consumo de oxígeno $\left(\mathrm{VO}_{2}\right)$, la glucosa y el ácido láctico, factores que eventualmente producen cambios cognitivos estables mediados por el ejercicio.

Para estudiar la actividad cerebral durante el ejercicio se han utilizado al menos dos técnicas, EEG y ERP. De acuerdo con Magnié, Bermon, Martin, Madany-Lounis, Suisse, Muhammad y Dolisi (2000), la actividad del EEG ha sido considerada como un indicador de la excitación cortical, mientras que P300, un componente de los ERP, indica la actividad neuronal relacionada con aspectos cognitivos básicos. Los ERP tienen dos componentes, la amplitud (i.e., $\mu \mathrm{V}$ ) y latencia (i.e., ms), las cuales representan la magnitud y la duración de la respuesta eléctrica al estímulo, respectivamente.

Recientemente, se ha utilizado la tomografía de emisión de positrones (positron emission tomography, PET, por sus siglas en inglés) en humanos como una técnica nuclear no invasiva para la cuantificación del flujo sanguíneo cerebral regional (regional cerebral blood flow, rCBF, por sus siglas en inglés) y metabolismo tisular in vivo (Iemitsu, Itoh, Fujimoto, Tashiro, Nagatomi, Ohmori, \& Ishii, 2000). Iemitsu et al. (2000) estudiaron el metabolismo de la glucosa en 12 sujetos sanos que corrieron 35 min a una intensidad del 70-75\% de su frecuencia cardiaca máxima predicha por la edad. Las mediciones posteriores al ejercicio indicaron que el consumo de glucosa cerebral durante el ejercicio fue estable en comparación con el dramático consumo en los músculos de las piernas. La principal preocupación metodológica en este estudio radicaba en que posiblemente la medición posterior al ejercicio realizada con el PET no reflejara fielmente el metabolismo cerebral durante el ejercicio; sin embargo, Iemitsu et al., indicaron que la acumulación de fluorodesoxiglucosa (FGD), una sustancia que se acumula en los tejidos, es rápida, y que en ese experimento sí reflejaba fielmente la actividad metabólica cerebral. Por lo tanto, la relevancia de este estudio radica en que el metabolismo cerebral se mantiene estable durante el ejercicio, a diferencia de otras regiones corporales en las que el flujo sanguíneo disminuye notablemente.

Se han encontrado resultados contradictorios acerca del efecto del ejercicio aeróbico sobre el flujo sanguíneo cerebral, el equilibrio y funcionamiento de los neurotransmisores, el tono neuroendocrino y autónomo (Ide \& Secher, 2000). Una posible causa de las divergencias pueda deberse al parámetro medido en los estudios. Por ejemplo, algunos investigadores miden el flujo sanguíneo cerebral global (global cerebral blood flow, gCBF, por sus siglas en inglés) y en otras ocasiones han medido el flujo sanguíneo cerebral regional (rCBF). De acuerdo con Ide y Secher, el gCBF y la tasa metabólica únicamente aumentan como resultado de la estimulación mental. Los investigadores sugieren que el rCBF sí cambia, sin embargo, el cambio varía dependiendo de la zona anatómica involucrada en el ejercicio. Por ejemplo, ya que las piernas tienen una pequeña representación en el homúnculo cerebral, los cambios podrían no ser tan dramáticos como los que ocurrirían en las manos o en el rostro. Esta respuesta ha sido descrita recientemente como la hipótesis hipofrontal transitoria (Dietrich \& Sparling, 2004), por medio de la cual tiene que 
ocurrir una activación masiva y sostenida de los sistemas motor y sensorial durante el ejercicio en detrimento de la actividad en otras estructuras neuronales. Por lo tanto, ocurre una inhibición temporal de las regiones cerebrales que no son esenciales para ejecutar el ejercicio específico.

El efecto del ejercicio aeróbico o del ejercicio contra resistencia sobre la actividad cerebral no se comprende plenamente. Con base en el modelo del aumento en el rCBF, la activación cortical asociada con movimientos dinámicos como ciclismo o carrera involucra las áreas sensorimotoras primarias y suplementarias. Por el contrario, durante contracciones estáticas o sostenidas, como por ejemplo cuando se levantan pesas, esas áreas podrían no activarse o solamente activarse parcialmente (Ide \& Secher, 2000).

Se ha mencionado que el ejercicio aeróbico afecta los ERP al promover un aumento en el flujo sanguíneo cerebral, con el concomitante aumento en el transporte y $\mathrm{VO}_{2}$, y la mejoría en el metabolismo de los neurotransmisores como la acetilcolina (ACh), dopamina (DOPA), norepinefrina (NE), y serotonina (SE) (Magnié et al., 2000). Ha habido una crítica a estudios similares debido al efecto confusor de algunas variables, por ejemplo, se ha mencionado que la hipertermia inducida por el ejercicio y la taquicardia podrían haber creado algún tipo de ruido ya que también se les asocia con respuestas P300.

En el estudio descrito por Magnié et al. (2000), se encontró que la latencia P300 en reposo se redujo en los adultos mayores con mejor condición física que en los que tenían mala condición física, y en adultos jóvenes luego de 3 min de ejercicio submáximo en cicloergómetro, el cual también mostró un aumento en la amplitud P300. Se piensa que la amplitud P300 se activa por un estímulo visual, y por consiguiente puede considerarse un estimador válido del procesamiento de la información visual.

El aumento en la respuesta de los P300 en la corteza visual también se puede relacionar con una mayor expresión genética (Jiaxu \& Weiyi, 2000). En un estudio realizado con ratas, Jiaxu y Weiyi (2000) midieron la expresión genética de la molécula proopiomelanocortina (POMC), un precursor de $\beta$-endorfinas y de la hormona adrenocorticotropina (ACTH), las cuales están involucradas en la regulación del eje hipotalámico-pituitario-adrenocortical (HPA). En el estudio, un grupo de ratas fueron ejercitadas $20 \mathrm{~min}$ en una banda sin fin durante siete semanas, y la velocidad de la banda durante el experimento cambió durante el transcurso del experimento (rango de 15-35 m/min). Después de este programa de ejercicio crónico, se encontró que la expresión del gen POMC cambió dependiendo del área cerebral estudiada. Por ejemplo, la expresión del ácido ribonucleico mensajero $\left(\mathrm{ARN}_{\mathrm{m}}\right)$ del gen POMC aumentó en la corteza frontal, pero no cambió en el hipocampo o en el hipotálamo. En conjunto, estos resultados indican tanto cambios estructurales como funcionales en las áreas cerebrales responsables del procesamiento visual y perceptual. Estos hallazgos son relevantes debido a que se ha postulado que las alteraciones en la expresión genética inducen cambios en el funcionamiento cerebral, lo cual podría considerarse como el mecanismo por medio del cual el ejercicio interviene en la evaluación de la imagen corporal (Kandel, 1998).

En un estudio similar llevado a cabo con ratas por McCloskey et al. (2001), se investigó la actividad de la enzima citocromo oxidasa (COX), una enzima mitocondrial involucrada en la producción aeróbica de la adenosina trifosfato (ATP). El diseño del estudio incluyó el entrenamiento voluntario de las ratas durante seis meses, en donde tenían que correr en una rueda unida a sus jaulas. Al final de la intervención experimental, las ratas habían alcanzado recorrer aproximadamente $6147 \mathrm{~m} /$ día, y se observó un aumento en la actividad de la enzima COX en comparación con el grupo control de ratas que no se ejercitaron. Se encontró el aumento en la actividad de la enzima 
COX en las patas y su representación en la corteza motora, lo que significa que se necesita un mayor metabolismo oxidativo para que ocurra una frecuencia mayor en la actividad neuronal asociado con correr a mayores velocidades (McCloskey et al., 2001).

En otros estudios en los que han utilizado cerdos, se han reportado aumentos significativos en el flujo sanguíneo en las regiones visuales durante el ejercicio (Delp, Armstrong, Godfrey, Laughlin, Ross, \& Wilkerson, 2001). En ese estudio los animales se ejercitaron durante 15 min al $70 \%$ y al 100\% de su consumo máximo de $\mathrm{O}_{2}\left(\mathrm{VO}_{2}\right.$ máx). Delp et al. (2001) reportaron una menor resistencia vascular al flujo sanguíneo a las áreas cerebrales responsables del procesamiento visual, contrario al aumento en la resistencia vascular al flujo en las áreas cerebrales responsables del olfato y la audición. La relevancia de estos hallazgos radica en que si se extrapolaran los resultados a los humanos, significaría que una persona podría reducir su capacidad para escuchar y oler durante una sesión aguda de ejercicios; sin embargo, aumentaría su capacidad de procesamiento visual.

\section{Ejercicio e imagen corporal}

En esta sección se discuten los efectos del ejercicio o el entrenamiento físico en la imagen corporal. El énfasis será en la descripción de la modalidad, frecuencia e intensidad de los programas de entrenamiento diseñados para mejorar la imagen corporal.

La imagen corporal, "la representación interna de su apariencia externa" (Hausenblas \& Symons, 2001, p. 323), identifica dos factores íntimamente relacionados; el "yo material" y el "yo somático" (Skrinar et al., 1986). Como se describió anteriormente, esta definición supone que la identificación de la imagen corporal es un proceso que requiere la evaluación perceptual (componente somático) de la apariencia externa (yo material).

Los estudios acerca del efecto del ejercicio físico sobre la imagen corporal son contradictorios. Una posible explicación por la que se puedan explicar dichos resultados es la definición de imagen corporal utilizada. Por ejemplo, los investigadores han utilizado diferentes definiciones del constructo de imagen corporal, como por ejemplo, atractivo físico (Anderson, Foster, McGuigan, Seebach, \& Porcari, 2004), o apariencia corporal (Skrinar et al., 1986), que podrían o no significar lo mismo.

Los estudios en los que se ha analizado el efecto de la modalidad del ejercicio (i.e., aeróbico vs. resistencia) en la imagen corporal han demostrado resultados positivos (Tucker \& Maxwell, 1992) o no han mostrado cambios (Anderson et al., 2004; Skrinar et al., 1986). Solamente en un estudio reciente se utilizó una combinación de ejercicios aeróbicos, contra resistencia y conferencias sobre fisiologia del ejercicio en estudiantes de secundaria masculinos y femeninos (Perry, Rosenblatt, Kempner, Feldman, Paolercio, \& Van Bemden, 2002). En comparación con sujetos control, la satisfaccion corporal mejoró en aquellos que se ejercitaron y recibieron conferencias sobre conceptos de salud.

La duración de los programas de entrenamiento ha variado de seis a 15 semanas (tabla 1). No en todos los estudios revisados se ha reportado la frecuencia de las sesiones de ejercicio. En la mayoría de los estudios en donde se ha utilizado el paradigma aeróbico, los participantes asistieron dos o tres veces por semana y se ejercitaron a intensidades entre el 40-80\% del $\mathrm{VO}_{2}$ máx; o en el rango del $60-75 \%$ de la frecuencia cardiaca máxima predicha. Asimismo, en los estudios donde se ha utilizado el paradigma de entrenamiento contra resistencia o de levantamiento de pesas, los participantes han realizado ejercicios en dos a tres series, y entre 10-15 repeticiones por serie. Con respecto a la duración de cada sesión, independientemente del paradigma utilizado ha variado de 20 a 45 minutos por sesión. 
Tabla 1

Resumen de los estudios acerca del efecto del ejercicio en la imagen corporal

\begin{tabular}{|c|c|c|}
\hline Referencia & Muestra & Intervención \\
\hline $\begin{array}{l}\text { Skrinar et } \\
\text { al. (1986) }\end{array}$ & $\begin{array}{l}\text { - } \quad 15 \text { mujeres } \\
\text { - } \quad 20-30 \text { años }\end{array}$ & $\begin{array}{l}\text { Ejercicio aeróbico } \\
\text { - Caminar de } 32 \text { a } 85 \mathrm{~km} / \text { semana } \\
\text { - } 8 \text { semanas, } 70-80 \% \text { VO2máx } \\
\text { - } \quad>3.5 \mathrm{~h} / \text { día, caminar, ciclismo, } \\
\text { etc }\end{array}$ \\
\hline $\begin{array}{l}\text { Tucker y } \\
\text { Maxwell } \\
(1992)\end{array}$ & $\begin{array}{l}\text { - } \quad 60 \text { mujeres } \\
\text { - } \quad 20 \text { años }\end{array}$ & $\begin{array}{l}\text { Ejercicios contra Resistencia (Pesas } \\
\text { - } 15 \text { semanas, } 2 \text { veces/semana } \\
\text { - } 40-45 \mathrm{~min} / \text { sesión } \\
\text { - } \quad 3 \text { series / } 10 \text { repeticiones / } \\
\text { ejercicio }\end{array}$ \\
\hline $\begin{array}{l}\text { Annesi } \\
(2000)\end{array}$ & $\begin{array}{l}\text { - } 13 \text { mujeres } \\
\text { - } \quad \text { Obesas } \\
\text { - } \quad \text { Sedentarias } \\
\text { - } 35 \text { años }\end{array}$ & $\begin{array}{l}\text { Aeróbico }+ \text { Pesas } \\
\text { - } 12 \text { semanas, } 2 \text { veces/semana } \\
\text { - Muy baja intensidad }(40-50 \% \\
\text { VO2máx) }\end{array}$ \\
\hline $\begin{array}{l}\text { Williams } \\
\text { y Cash } \\
(2001)\end{array}$ & $\begin{array}{ll}\text { - } & 27 \text { mujeres } \\
\text { - } & 12 \text { hombres } \\
\text { - } & 21.7 \text { años }\end{array}$ & $\begin{array}{l}\text { Pesas } \\
\text { - } \quad 6 \text { semanas, } 3 \mathrm{~h} / \text { semana }\end{array}$ \\
\hline
\end{tabular}


Tabla 1 (continuación)

\begin{tabular}{|c|c|c|c|}
\hline $\begin{array}{l}\text { Ahmed et } \\
\text { al. (2002) }\end{array}$ & $\begin{array}{ll}\text { - } & 49 \text { mujeres } \\
\text { - } & 20 \text { años }\end{array}$ & $\begin{array}{l}\text { Pesas } \\
\text { - } 12 \text { semanas, } 2 \text { veces/semana, } 40 \\
\text { min/sesión }\end{array}$ & $\begin{array}{l}\text { - } 51 \% \text { indicaron una } \\
\text { imagen corporal positiva } \\
\text { como resultado del } \\
\text { programa. } \\
\text { Diseño del estudio débil. } \\
\text { Solamente se midió la } \\
\text { imagen corporal después } \\
\text { del finalizar el programa } \\
\text { de ejercicios. }\end{array}$ \\
\hline $\begin{array}{l}\text { Perry et al. } \\
(2002)\end{array}$ & $\begin{array}{ll}\text { - } & 161 \\
& \text { estudiantes } \\
\text { - } & \text { Secundaria } \\
\text { - } & 87 \text { mujeres } \\
\text { - } & 74 \text { hombres } \\
\text { - } & 16.5 \text { años }\end{array}$ & $\begin{array}{l}\text { Combinación } \\
\text { Aeróbico: } \\
\text { - } 40-45 \mathrm{~min} / \mathrm{semana}, 60-75 \% \\
\text { frecuencia cardiaca máxima } \\
\text { predicha } \\
\text { - } \quad \text { Pesas: } \\
\text { - } 20-30 \mathrm{~min} / \mathrm{semana}, 8-12 \\
\text { repeticiones máximas } \\
\text { - Conferencias de fisiología del } \\
\text { - ejercicio } \\
\text { Clases magistrales }\end{array}$ & $\begin{array}{l}\text { - Mejorías en la } \\
\text { satisfacción con el } \\
\text { cuerpo en comparación } \\
\text { con sujetos control. }\end{array}$ \\
\hline $\begin{array}{l}\text { Anderson } \\
\text { et al. } \\
(2004)\end{array}$ & $\begin{array}{l}\text { - } 28 \text { hombres } \\
\text { - } \quad \text { Sedentarios } \\
18-40 \\
\text { años }\end{array}$ & $\begin{array}{l}\text { Aeróbico } \\
\text { - } 6 \text { semanas, } 3 \mathrm{~d} / \mathrm{semana}, 20 \mathrm{~min} / \\
\text { sesión } \\
\text { - } 60-80 \% \mathrm{VO} 2 \mathrm{máx} \\
\text { - } \quad \text { Pesas } \\
\text { - } 6 \text { semanas, } 3 \mathrm{~d} / \mathrm{semana}, 30 \mathrm{~min} / \\
\text { sesión } \\
2 \text { series/ejercicio, } 10-15 \\
\text { repeticiones/serie }\end{array}$ & $\begin{array}{l}\text { No hubo diferencias } \\
\text { entre grupos en el } \\
\text { atractivo físico. }\end{array}$ \\
\hline
\end{tabular}

Finalmente, en un reciente meta-análisis, Hausenblas y Symons (2001), analizaron 78 investigaciones para determinar si los atletas tenían una imagen corporal diferente a las personas que no realizan ejercicio. Los investigadores calcularon 294 tamaños de efecto (TE), una medida estandarizada para estimar la magnitud del efecto del ejercicio en la imagen corporal, y encontraron que los atletas reportaron mejores percepciones de su imagen corporal que las personas que no realizaban ejercicio. El tamaño del efecto encontrado fue consistentemente bajo $(T E=0.27)$, aún después de ajustar los valores tomando en cuenta el índice de masa corporal, el tipo de deporte, el sexo y la edad de los sujetos. En general, este hallazgo significa que la imagen corporal es consistentemente percibida de manera positiva entre diversos grupos de atletas o en personas que se mantienen físicamente activas. 


\section{Conclusiones}

La evidencia presentada por Goodale (2001) y Norhoff (2001) apoyan el supuesto que indica que la corteza parietal derecha es la estructura responsable de la generación de la imagen propia del cuerpo, mientras que la corteza parietal izquierda juzga o compara el cuerpo con otros cuerpos, lo que da como resultado una representación holística de la imagen corporal.

Con base en los estudios preliminares de Downing et al. (2001), ACE es la estructura cerebral aparentemente responsable del procesamiento de la información para generar una imagen corporal; sin embargo, se requiere mucha mayor investigación en humanos al utilizar técnicas como fMRI, una técnica no invasiva que proporciona información valiosa acerca de la actividad eléctrica del cerebro. Se espera que ACE sea una estructura que muestre una notable plasticidad ante los constantes estímulos mecánicos y perceptuales (e.g., visual, táctil), tal y como podría proporcionarlo el ejercicio físico.

La mayoría de investigaciones apoyan los cambios plásticos y funcionales en el cerebro que ocurren como resultado del ejercicio físico. Los cambios plásticos incluyen la expresión genética y supervivencia y proliferación celular (Jiaxu \& Weiyi, 2000; McCloskey et al., 2001; Yu et al., 2003), mientras que los cambios funcionales involucran la actividad metabólica (Iemitsu et al., 2000; Ide \& Secher, 2000; Magnié et al., 2000; McCloskey et al., 2001). Con base en estos estudios, se concluye que el flujo sanguíneo cerebral se mantiene constante durante el ejercicio a pesar de una marcada redistribución hacia otras áreas responsables del procesamiento de la información visual. Se sugiere que el efecto del ejercicio dinámico (e.g., correr, ciclismo) en la corteza visual podría ser diferente al que produciría el ejercicio estático (e.g., levantar pesas, entrenamiento contra resistencia) ya que la representación cortical de las áreas involucradas producirían respuestas metabólicas y hemodinámicas diferentes (Dalsgaard, Voliantis, Yoshiga, Dawson, \& Secher, 2004). El potencial impacto del ejercicio en un constructo tan complejo como la imagen corporal es muy limitado y claramente especulativo en este momento. Todavía hace falta más investigación que sustente los potenciales cambios neurológicos, genéticos y que estudie los cambios en la distribución del flujo sanguíneo, tanto en modelos animales como en modelos humanos. Probablemente el aspecto cognitivo de la intervención podría explicar una proporción de la variancia del cómo deberíamos comprender el constructo de la imagen corporal; sin embargo, se necesitan más investigaciones para apoyar esta hipótesis.

En grupos de personas físicamente activas y en atletas se ha encontrado una imagen corporal positiva. Sin embargo, estudios individuales han mostrado claras inconsistencias en la definición del término imagen corporal, en la modalidad, la frecuencia e intensidad del ejercicio ejecutado, así como en los instrumentos de medición utilizados para medir el constructo. Más aun, de acuerdo con Stunkard (2000), existen al menos 22 instrumentos para medir la imagen corporal. La mayoría de estudios carecen de un sustento científico serio en el que se expliquen mecanismos fisiológicos, y tampoco se han llevado a cabo estudios de seguimiento cuando se han encontrado resultados favorables en la percepción de la imagen corporal.

A pesar de estas limitaciones, la investigación en el área de la imagen corporal es promisoria. Las nuevas tecnologías pueden ayudar a comprender mejor los cambios cerebrales en respuesta a sesiones agudas y crónicas de ejercicio que llevan, a su vez, a redefinir nuestra imagen corporal. Parece que el ejercicio aeróbico consistentemente cambia la estructura y funcionamiento cerebral al proporcionar una mayor cantidad de sangre y nutrientes a las áreas responsables del procesamiento 
visual. En este momento, no existe información relacionada al efecto del entrenamiento contra resistencia en los cambios en estructura y funcionamiento cerebral.

De acuerdo con Branch (1998), el ejercicio vibratorio podría cambiar la imagen corporal al crear una ilusión en que la persona percibe sus brazos más largos de lo que realmente son. Este es un simple ejemplo de cómo el cerebro recibe e interpreta los diferentes estímulos sensoriales. A pesar de ello, el tema de la imagen corporal es complejo y no puede explicarse de una manera reduccionista utilizando únicamente factores biológicos o psicológicos. Por lo tanto, se necesitan modelos multidimensionales, en los que se incluya al menos aspectos sociales, psicológicos y biológicos para comprender cómo valoramos nuestra imagen corporal.

\section{Referencias}

Ahmed, C., Hilton, W., \& Pituch, K. (2002). Relationship of strength training to body image among a sample of female university students. Journal of Strength and Conditioning Research, 16(4), 645-648.

Anderson, M. L., Foster, C., McGuigan, M . R., Seebach, E., \& Porcari, J. P. (2004). Training vs. body image: Does training improve subjective appearance ratings? Journal of Strength and Conditioning Research, 18(2), 255-259.

Annesi, J. J. (2000). Effects of minimal exercise and cognitive behavior modification on adherence, emotion change, self-image, and physical change in obese women. Perceptual and Motor Skills, 91(1), 322-336.

Berlucchi, G. \& Aglioti, S. (1997). The body in the brain: neural bases of corporal awareness. Trends in Neuroscience, 20(12), 560-564.

Branch, H. (1998). Evidence for a disturbance of the body schema in neglect. Brain and Cognition, 37, 527-544.

Bryan, A. D. \& Rocheleau, C. A. (2002). Predicting aerobic versus resistance exercise using the Theory of Planned Behavior. American Journal of Health Behavior, 26(2), 83-94.

Buckworth, J. \& Dishman, R. K. (2002). Exercise psychology. Champaign, IL: Human Kinetics.

Cash, T. F. \& Deagle, E. A. III. (1997). The nature and extent of body image disturbances in anorexia nervosa and bulimia nervosa: A meta-analysis. International Journal of Eating Disorders, 22(2), 107-125.

Chen, L. M., Friedman, R. M., \& Roe, A. W. (2003). Optical imaging of a tactile illusion in area 3b of the primary somatosensory cortex. Science, 302(5646), 881-885.

Cohen, J. D. \& Tong, F. (2001). The face of controversy. Science, 293(5539), 2405-2407.

Coslett, H. B. (1998). Evidence for a disturbance of the body schema in neglect. Brain and Cognition, 37(3), 527-544.

Dallow, C. B. \& Anderson, J. (2003). Using Self-efficacy and a Transtheoretical Model to develop a physical activity intervention for obese women. American Journal of Health Promotion, 17(6), 373-382.

Dalsgaard, M. K., Voliantis, S., Yoshiga, C. C., Dawson, E. A., \& Secher, N. H. (2004). Cerebral metabolism during upper and lower body exercise. Journal of Applied Physiology, 97(5), 1733-1739. 
Deforche, B., Bourdeaudhuij, I., Tanghe, A., Hills, A., \& De Bode, P. (2004). Changes in physical activity in children and psychosocial determinants of physical activity in children and adolescents treated for obesity. Patient Education and Counseling, 55(3), 407-415.

Delp, M. D., Armstrong, R. B., Godfrey, D. A., Laughlin, M. H., Ross, C. D., \& Wilkerson, M. K. (2001). Exercise increases blood flow to locomotor, vestibular, cardiorespiratory and visual regions of the brain in miniature swine. Journal of Physiology, 533(3), 849-859.

Dietrich, A. \& Sparling, P. B. (2004). Endurance exercise selectively impairs prefrontal-dependent cognition. Brain and Cognition, 55(3), 516-524.

Dishman, R. K., Motl, R. W., Saunders, R., Felton, G., Ward, D. S., Dowda, M., \& Pate, R. R. (2004). Self-efficacy partially mediates the effect of a school-based physical-activity intervention among adolescent girls. Preventive Medicine, 38(5), 628-636.

Dohle, C., Kleiser, R., Seitz, R. J., \& Freund, H. J. (2004). Body scheme gates visual processing. Journal of Neurophysiology, 91, 2376-2379.

Downing, P. E., Jiang, Y., Shuman, M., \& Kanwisher, N. (2001). A cortical area selective for visual processing of the human body. Science, 293(5539), 2470-2473.

Ehrsson, H. H., Spence, C., \& Passingham, R. E. (2004). That's my hand! Activity in the premotor cortex reflects feeling of ownership of a limb. Science, 305(5685), 875-877.

Elbert, T. \& Rockstroh, B. (2004). Reorganization of the human cerebral cortex: The range of changes following use and injury. The Neuroscientist, 10(2), 129-141.

Evans, R. W. (2004). The Alice in Wonderland Syndrome. Headache, 44, 624-625.

Eysel, U. T. (2003). Illusions and perceived images in the primate brain. Science, 302(5646), 789790.

Feingold,A. \& Mazzella, R. (1998). Gender differences in body image are increasing. Psychological Science, 9(3), 190-195.

Gokee-La Rose, J., Dunn, M. E., \& Tantleff-Dunn, S. (2004). An investigation of the cognitive organization of body comparison sites in relation to physical appearance related anxiety and drive for thinness. Eating Behaviors, 5, 133-145.

Goodale, M. A. (2001). Different spaces and different times for perception and action. Progress in Brain Research, 134, 313-331.

Hargreaves, D. A. \& Tiggemann, M. (2004). Idealized media images and adolescent body image: "comparing" boys and girls. Body Image, 1(4), 351-361.

Hari, R., Hänninen, R., Mäkinen, T., Jousmäki, V., Forss, N., Seppä, M., \& Salonen, O. (1998). Three hands: fragmentation of human bodily awareness. Neuroscience Letters, 240(3), 131-134.

Hausenblas, H. A. \& Symons, D. (2001). Comparison of body image between athletes and nonathletes: A meta-analytic review. Journal of Applied Sport Psychology, 13, 323-339.

Helmuth, L. (2002). Redrawing the brain's map of the body. Science, 296(5573), 1587-1588.

Ide, K. \& Secher, N. H. (2000). Cerebral blood flow and metabolism during exercise. Progress in Neurobiology, 61(4), 397-414. 
Iemitsu, M., Itoh, M., Fujimoto, T., Tashiro, M., Nagatomi, R., Ohmori, H., \& Ishii, K. (2000). Whole-body energy mapping under physical exercise using positron emission tomography. Medicine and Science in Sports and Exercise, 32(12), 2067-2070.

Jeannerod, M. (2004). Visual and action cues contribute to the self-other distinction. Nature Neuroscience, 7(5), 422-423.

Jenkins, W. M., Merzenich, M. M., Ochs, M. T., Allard, T., \& Guic-Robles, E. (1990). Functional reorganization of primary somatosensory cortex in adult owl monkeys after behaviorally controlled tactile stimulation. Journal of Neurophysiology, 63(1), 82-104.

Jiaxu, C. \& Weiyi, Y. (2000). Influence of acute and chronic treadmill exercise on rat brain POMC gene expression. Medicine and Science in Sports and Exercise, 32(5), 954-957.

Kandel, E. R. \& Mack, S. (2003). A parallel between radical reductionism in science and in art. Annals of the New York Academy of Sciences, 1001, 272-294.

Kandel, E. R. (1998). A new intellectual framework for psychiatry. American Journal of Psychiatry, 155(4), 457-469.

Katzmarzyk, P. T. \& Davis, C. (2001). Thinness and body shape of Playboy centerfolds from 1978 to 1998. International Journal of Obesity, 25, 590-592.

Kirkcaldy, B. D., Shephard, R. J., \& Siefen, R. G. (2002). The relationship between physical activity and self-image and problem behaviour among adolescents. Social Psychiatry and Psychiatric Epidemiology, 37(11), 544-550.

Magnié, M. N., Bermon, S., Martin, F., Madany-Lounis, M., Suisse, G., Muhammad, W., \& Dolisi, C. (2000). P300, N400, aerobic fitness, and maximal aerobic exercise. Psychophysiology, 37(3), 369-377.

Martin, E., Thiel, T., Joeri, P., Loenneker, T., Ekatrodramis, D., Huisman, T., Henning, J., \& Marcar, V. L. (2000). Effect of pentobarbital on visual processing in man. Human Brain Mapping, 10(3), 132-139.

Mayville, S., Katz, R. C., Gipson, M. T., \& Cabral, K. (1999). Assessing the prevalence of body dysmorphic disorder in an ethnically diverse group of adolescents. Journal of Child and Family Studies, 8(3), 357-362.

McCloskey, D. P., Adamo, D. S., \& Anderson, B. J. (2001). Exercise increases metabolic capacity in the motor cortex and striatum, but not in the hippocampus. Brain Research, 891(1-2), 168-175.

McLaren, L. \& Kuh, D. (2004). Body dissatisfaction in midlife women. Journal of Women \& Aging, 16(1/2), 35-53.

Netz, Y. \& Raviv, S. (2004). Age differences in motivational orientation towards physical activity: An application of the social-cognitive theory. The Journal of Psychology, 138(1), 35-49.

Northoff, G. (2001). "Brain-paradox" and "embeddment" - Do we need a "philosophy of the brain"? Brain and Mind, 2(2), 195-211.

Pate, R. R., Pratt, M., Blair, S. N., Haskell, W. L., Macera, C. A., Bouchard, C., Buchner, D., Ettinger, W., Heath, G., King, A. C., Kriska, A. M., Leon, A. S., Marcus, B. H., Morris, J., Paffenbarger, R. S., Patrick, K., Pollock, M. L., Rippe, J. M., Sallis, J. F., \& Wilmore, 
J. H. (1995). Physical activity and public health: A recommendation of the Centers for Disease Control and Prevention and the American College of Sports Medicine. Journal of the American Medical Association, 273(5), 402-407.

Perry, A. C., Rosenblatt, E. S., Kempner, L., Feldman, B. B., Paolercio, M. A., \& Van Bemden, A. L. (2002). The effects of an exercise physiology program on physical fitness variables, body satisfaction, and physiology knowledge. Journal of Strength and Conditioning Research, 16(2), 219-226.

Pompili, M., Mancianelli, I., Girardi, P., Ruberto, A., \& Tatarelli, R. (2004). Suicide in anorexia nervosa: A meta-analysis. International Journal of Eating Disorders, 36(1), 99-103.

Press, C., Taylor-Clarke, M., Kennett, S., \& Haggard, P. (2004). Visual enhancement of touch in spatial body representation. Experimental Brain Research, 154(2), 238-245.

Rovniak, L. S., Anderson, E. S., \& Winett, R. A. (2002). Social cognitive determinants of physical activity in young adults: A prospective structural equation analysis. Annals of Behavioral Medicine, 24(2), 149-156.

Scully, D., Kremer, J., Meade, M. M., Graham, R., \& Dudgeon, K. (1998). Physical exercise and psychological well being: a critical review. British Journal of Sports Medicine, 32(2), 111120.

Schultes, R. E. \& Hofmann, A. (1979). Plants of the Gods: Origins of Hallucinogenic Use. Maidenhead, England: McGraw-Hill.

Seeger, G., Braus, D. F., Ruf, M., Goldberger, U., \& Schmidt, M. H. (2002). Body image distortion reveals amygdala activation in patients with anorexia nervosa - a functional magnetic resonance imaging study. Neuroscience Letters, 326(1), 25-28.

Skrinar, G. S., Bullen, B. A., Cheek, J. M., Mc Arthur, J. W., \& Vaughan, L. K. (1986). Effects of endurance training on body-consciousness in women. Perceptual and Motor Skills, 62(2), 483-490.

Stekelenburg, J. J. \& de Gelder, B. (2004). The neural correlates of perceived human bodies: an ERP study on the body-inversion effect. NeuroReport, 15(5), 777-780.

Stunkard, A. (2000). Old and new scales for the assessment of body image. Perceptual and Motor Skills, 90(3), 930.

Taub, E., Uswatte, G., \& Elbert, T. (2002). New treatments in neurorehabilitation founded on basic research. Nature Reviews Neuroscience, 3(3), 228-236.

Taveras, E. M., Rifas-Shiman, S. L., Field, A. E., Frazier, A. L., Golditz, G. A., \& Gillman, M. W. (2004). The influence to look like media figures on adolescent physical activity. Journal of Adolescent Health, 35(1), 41-50.

Trost, S. G., Kerr, L. M., Ward, D. S., \& Pate, R. R. (2001). Physical activity and determinants of physical activity in obese and non-obese children. International Journal of Obesity, 25(6), 822-829.

Trost, S. G., Saunders, R., \& Ward, D. S. (2002). Determinants of physical activity in middle school children. American Journal of Health Behavior, 26(2), 95-102. 
Tucker, L. A. \& Maxwell, K. (1992). Effects of weight training on the emotional well-being and body image of females: Predictors of greatest benefit. American Journal of Health Promotion, 6(5), 338-344.

Umaña, M. (2005, Febrero 13). Obsesión por tener unos kilos menos. Periodico Al Dia. Recuperado de http://www.aldia.co.cr/ad_ee/2005/febrero/13/nacionales0.html

United States Department of Health and Human Services. (1996). Physical activity and health: A report of the Surgeon General. Atlanta, GA: U.S. Department of Health and Human Services, Centers for Disease Control and Prevention, National Center for Chronic Disease Prevention and Health Promotion.

Vogeley, K., Kurthen, M., Falkai, P., \& Maier, W. (1999). Essential functions of the human self model are implemented in the prefrontal cortex. Consciousness and Cognition, 8(3), 343363.

Wagner, A., Ruf, M., Braus, D. F., \& Schmidt, M. H. (2003). Neuronal activity changes and body image distortion in anorexia nervosa. NeuroReport, 14(17), 2193-2197.

Williams, P. A. \& Cash, T. F. (2001). Effects of a circuit weight training program on the body images of college students. International Journal of Eating Disorders, 30(1), 75-82.

Yu, B. K., Yoon, B. C., Kim, S. S., Chung, S. L., Kim, E. H., Kim, K. M., Lim, B. V., Jang, M. H., Chung, J. H., \& Kim, C. J. (2003). Treadmill exercise increases cell proliferation in hippocampal dentate gyrus in alcohol-intoxicated rats. Journal of Sports Medicine and Physical Fitness, 43(3), 393-397.

\section{Reseña del autor}

\section{José Moncada Jiménez}

Catedrático Universidad de Costa Rica. Doctor en Ciencias (Ph.D.), énfasis en ciencias biomédicas. Graduado de la Universidad de Costa Rica. Labora actualmente en la Escuela de Educación Física y Deportes de la Universidad de Costa Rica. Dirije el Laboratorio de Ciencias del Movimiento Humano. 